\title{
Postpartum Depression and Risk Factors among Vietnamese Women
}

\author{
Thi Kim Ly Do ${ }^{1}$, ${ }^{1}$ Thi Thanh Huong Nguyen, ${ }^{2}$ and Thi Thu Huong Pham ${ }^{2}$ \\ ${ }^{1}$ Advanced Nursing Program, Hanoi Medical University, Vietnam \\ ${ }^{2}$ Faculty of Nursing and Midwifery, Hanoi Medical University, Vietnam \\ Correspondence should be addressed to Thi Kim Ly Do; dothikimly95@gmail.com
}

Received 3 May 2018; Accepted 29 August 2018; Published 18 September 2018

Academic Editor: Roger Ho

Copyright (c) 2018 Thi Kim Ly Do et al. This is an open access article distributed under the Creative Commons Attribution License, which permits unrestricted use, distribution, and reproduction in any medium, provided the original work is properly cited.

Postpartum depression (PPD) places a burden on maternal health. PPD exerts a negative impact on mothers' health and children's life. The purpose of this research was to identify the prevalence of PPD and the risk factors contributing to PPD. Therefore, a crosssectional quantitative study was conducted. 116 women were categorized into two groups. One category included new mothers who received scores of Edinburgh Postpartum Depression Scale (EPDS) 12 or more. The other category included mothers who received scores less than 12. Descriptive statistic and then binary logistic regression were also performed. For EPDS $\geq 12$, the prevalence of PPD was $27.6 \%$ among new mothers during the first year after delivery. Level of education, diseases during pregnancy, being the first-time mothers, dissatisfaction about family, and limited communication and interaction with others were significant predictors of PPD.

\section{Background}

Postpartum depression (PPD) is a major maternal health problem in the first year after giving birth. According to a study published in the British Journal of Psychiatry in 2017, the prevalence of PPD was $13-40 \%$ [1]. A study conducted in Vietnam indicated that the prevalence of PPD among new mothers was $20.4 \%$ in urban areas and was $15.8 \%$ in rural areas [2]. Previous studies have suggested that the mother's socioeconomic status and living the different areas create different prevalence of PPD [3]. Mothers living in poverty are more likely to be depressed and have greater barriers to access to treatment than the general population [4]. A research was conducted in Vietnam, indicated that 58\% that seriously ill low-income patients who face higher health care costs would quit their treatment [5]. Depression is treatable but costly, so influencing quality of life of people [6]. Particularly in Vietnam healthcare system, the probability of satisfaction conditional on insurance reimbursement is lower for patients with residency status and higher for those without [7]. Nontreatment or nondiagnosis of PPD can have an adverse long-term effect [8]. Postpartum depression is associated with reducing the physical and mental health of women's lives [9].
PPD may also lead to lower cognitive and linguistic development in the first year of life for the child(ren) [10], and they might experience behavioral disorder and impair physical development $[11,12]$. Suicide is known to be one of the worst consequences of PPD [13, 14]. Psychoses occur in 1 to 2 per 1000 postpartum women; they may present as schizophrenic or affective disorder or as confusional states $[15,16]$. Numerous studies have demonstrated many factors associated with PPD including obstetric history, mode of delivery [17], biochemical genetic [18, 19], and other social stressors: age [20]; socioeconomy [21, 22]; culture [23]; education; negative life events. To prevent, early treatment of PPD is likely to reduce public sector costs, increase earnings, and improve quality of life for women [24].

\section{Method}

This was a cross-sectional study conducted from August 20 to September 7 in Hanoi. This study will include all women whose child(ren) are less 1 year old and they are over 18 years old. 116 postpartum women were screened for postpartum depression using Edinburgh Postpartum Depression Scale 
TABle 1: Demographic.

\begin{tabular}{llcc}
\hline & Variable & Number (N=116) & Percentage (\%) \\
\hline \multirow{2}{*}{ Score of EPDS } & $\geq 12$ & 32 & 27.6 \\
& $<12$ & 84 & 72.4 \\
\hline \multirow{2}{*}{ Age } & $\leq 24$ & 30 & 25.9 \\
& $>24$ & 86 & 74.1 \\
\hline \multirow{2}{*}{ Education } & High school graduate and lower & 47 & 40.5 \\
& College and higher & 69 & 59.5 \\
\multirow{2}{*}{ Address } & Rural & 66 & 56.9 \\
& Urban & 50 & 43.1 \\
\hline \multirow{2}{*}{ Religion } & Yes & 13 & 103 \\
& No & & 88.8 \\
\hline
\end{tabular}

(EPDS). The EPDS includes 10 items measured in a Likert scale of $0-3$. Score of 12 and above indicates risk factors of depression. The sensitivity and specificity of EPDS were $65-100 \%$ and $49-100 \%$, respectively [25]. EPDS has been translated and validated for non-English speaking population $[26,27]$ and has used widely in Asian $[28,29]$. New mothers were also asked to report information about demographics, pregnancy and disease history, interpersonal skill, and characteristics.

Prior to the main survey, 10 new mothers were asked for a pilot interview to check for readability and clarity of the questionnaire. The face-to-face interview was conducted at participant's private house.

SPSS software version 23.0 was employed for data management and analysis. Descriptive statistic and then binary logistic regression were also performed.

The study was approved by ethics committee of Hanoi Medical University, Decision No. 5042 on November 7, 2017.

\section{Results}

A score of EPDS cut-off was $\geq 12$ and the prevalence of postpartum depression among 116 new mothers (18-43 years old) during the first year after childbirth was $27.6 \%$ (Table 1 ).

The majority of women were over 25 years old and graduated from college or higher $(\mathrm{N}=116,59.5 \%)$. There are 66 women who lived in rural areas (56.9\%) and 50 women who lived in urban areas $(43.1 \%)$. In this study, most people are nonreligious $(88.8 \%$ ) and just $11.2 \%$ have religions such as Buddhism, Christianty, and others.

(1) Chronic diseases including diabetes, cardiovascular diseases, hypotension, hypertension, anemia, Parkinson, Lupus immunity disorder, and cancer.

(2) Diseases during pregnancy including diabetes, hypertension/hypotension, hepatitis, chicken pox, and gynecological inflammation.

(3) Life-event including family member died or critical diseases, accident, and unfavorable condition.

An increased risk of maternal depressive symptoms was remarkably associated with the level of education (OR 0.4, $\mathrm{p}=0.036$ ). The odd ratio for this variable, however, is 0.4 , a value less than 1 . This indicates that new mothers with a lower level of education were less likely to experience PPD. The diseases during pregnancy contracted by the mother are also a significant predictor, according to sig. value $(\mathrm{p}=0.009)$. In the logistic regression model, the odd ratio of new mothers without gynecological diseases during pregnancy was 0.3 times less than likely to those experiencing diseases during pregnancy. Another variable in Table 2 found to be associated with the PPD's symptoms was whether they were first-time mothers. This indicated that those who became first-time mothers were over 2 times more likely to suffer from PPD than those who already had children.

Our results found that the variables for increasing EPDs were the satisfaction of new mothers about family life $(\mathrm{p}<$ 0.001). Postpartum women who were feeling happy were 0.2 times less likely to present with symptoms of PPD than the mother having arguments in their family. New mothers with limited communication and interaction with others were over 4 times more than the reference categories $(\mathrm{p}=0.001, \mathrm{OR} 4.4)$.

\section{Discussion}

Postpartum depression (PPD) is a major maternal health problem in the first year after giving birth. The prevalence of postpartum depression was $27.6 \%$ as measured by the Edinburgh Postpartum Depression Scale (EPDS) with a cutoff point $\geq 12$. Our study found the incidence of PPD was higher than it was estimated in Thua Thien Hue Province (18.1\%) [2] and Da Nang (19.3\%) [30]. Many studies have reported PPD rates from 18 countries [31]; the average lifetime and 12-month prevalence estimates of major depression were $14.6 \%$ and $5.5 \%$ in high-income countries and $11.1 \%$ and $5.9 \%$ in low- to middle-income countries. The result of our study did not correspond to some researches about the relationship between PPD and regional differences (rural areas and urban areas). These differences may be related to the mothers' characteristics (demographic, pregnant history, and interpersonal characteristics) and study design (sample, method, and cut-off point).

In our study, women with gestational diseases are at higher risk factor of PPD compared to women who have not. The causes of the influence of diseases (gestational diabetes, gynecological inflammation, hypertension or hypotension, 
TABLE 2: Factors associated with PPD.

\begin{tabular}{|c|c|c|c|c|c|c|}
\hline \multirow{2}{*}{ Variables } & & \multicolumn{2}{|c|}{ EPDS } & \multirow{2}{*}{ 95\% CI } & \multirow{2}{*}{ OR } & \multirow{2}{*}{ p-value } \\
\hline & & $\geq 12$ & $<12$ & & & \\
\hline \multicolumn{7}{|c|}{ Demographic factors } \\
\hline \multirow[t]{2}{*}{ Age } & $\leq 24$ & 8 & 22 & \multirow{2}{*}{$0.4-2.4$} & 0.9 & \multirow{2}{*}{0.89} \\
\hline & $>24$ & 24 & 62 & & Refer & \\
\hline \multirow[t]{2}{*}{ Education } & High school graduate and lower & 8 & 39 & \multirow{2}{*}{$0.2-0.9$} & 0.4 & \multirow{2}{*}{0.04} \\
\hline & College and higher & 24 & 45 & & Refer & \\
\hline \multirow[t]{2}{*}{ Address } & Rural & 16 & 50 & \multirow{2}{*}{$0.3-1.5$} & 0.7 & \multirow{2}{*}{0.35} \\
\hline & Urban & 16 & 34 & & Refer & \\
\hline \multirow[t]{2}{*}{ Religion } & Yes & 2 & 11 & \multirow{2}{*}{$0.5-10.8$} & Refer & \multirow{2}{*}{0.29} \\
\hline & No & 30 & 73 & & 2.3 & \\
\hline \multicolumn{7}{|c|}{ Pregnant history } \\
\hline \multirow[t]{2}{*}{ Chronic disease (1) } & Yes & 7 & 12 & \multirow{2}{*}{$0.2-1.7$} & Refer & \multirow{2}{*}{0.32} \\
\hline & No & 25 & 72 & & 0.6 & \\
\hline \multirow[t]{2}{*}{ Gynecological diseases during pregnancy (2) } & Yes & 11 & 11 & \multirow{2}{*}{$0.1-0.8$} & Refer & \multirow{2}{*}{0.01} \\
\hline & No & 21 & 73 & & 0.3 & \\
\hline \multirow[t]{2}{*}{ First-time mother } & Yes & 22 & 39 & \multirow{2}{*}{$1.1-6.0$} & 2.5 & \multirow{2}{*}{0.03} \\
\hline & No & 10 & 45 & & Refer & \\
\hline \multirow[t]{2}{*}{ Breastfeeding } & Yes & 31 & 80 & $0.2-14.4$ & 1.6 & 0.69 \\
\hline & No & 1 & 4 & & Refer & \\
\hline Planned pregnancy & Planned & 25 & 76 & $0.1-1.1$ & 0.4 & 0.07 \\
\hline & Unplanned & 7 & 8 & $0.1-1.1$ & Refer & 0.08 \\
\hline & Interpersonal characteristics & & & & & \\
\hline Family structure & Extended & 21 & 47 & $0.6-35$ & 1.5 & 0.34 \\
\hline & Nuclear & 11 & 37 & 8.05. & Refer & (0.0. \\
\hline Satisfaction about family life & Feeling happy & 8 & 55 & $0.1-0.4$ & 0.2 & 0.000 \\
\hline & Having arguments & 24 & 29 & & Refer & \\
\hline Communicate and interact with other & Limited than before & 23 & 31 & $1.8-10.6$ & 4.4 & 0.001 \\
\hline & As before & 9 & 53 & & Refer & \\
\hline Tell with others when struggling & Yes & 5 & 4 & $0.9-14.8$ & 3.7 & 0.05 \\
\hline & No & 27 & 80 & & Refer & \\
\hline Having stressful life-events (3) & Yes & 23 & 67 & $0.2-1.6$ & Refer & 0.36 \\
\hline & No & 9 & 17 & & 0.6 & \\
\hline
\end{tabular}

and hepatitis) on increasing PPD are not clear [32, 33]. It is possible that the diseases in pregnancy seem to be a psychological burden for women, with remarkable effects on developing PPD. For instance, one of some studies reviewed of the literature regarding gestational diabetes and PPD suggests that the percentage of women reporting PPD following a diagnosis of gestational diabetes was higher than women who did not [34].

Two strong predictors of developing PPD in this study were limited communication/interaction with others when struggling $(p=0.001)$ and dissatisfaction about their family life $(\mathrm{p}<0.001)$. Happiness and satisfaction with their life may prevent the development of PPD. Some theories explained the mechanism of emotional associated with PPD's development, "including the monaamine, biorhym, neuroendocrine, neuroimmune, and kindling/neuroplasticity theories" [35]. Many researchers hold the belief that the hormones (endorphin, serotonin, and dopamine) are released when having positive thinking and emotion $[18,36]$. Women who communicate and interact with others during the postpartum period are at a lower risk of increasing PPD. The previous studies described relationship between stress and limited relationship, as well as new mothers who do not want to communicate and share something with others $[8,37]$. The results from many researchers are similar to our findings.

However, the study was conducted in a short time (from August 20 to September 7, 2017) and a small sample size $(\mathrm{N}=116)$. Stigma, perceptions of motherhood, religion, and culture sensitively were major barriers to screening for PPD.

\section{Conclusion}

This study makes important contributions to knowledge about the prevalence of PPD in Hanoi and the risk factors associated with PPD. The prevalence of PPD during the first year after childbirth was $27.6 \%$. PPD is considered as a burden in the community. Several therapies are recommended to reduce the prevalence of PPD: conventional face-to-face 
therapy [38] (the range of pharmacological, psychotherapeutic, and other nonpharmacologic interventions); a possibility of mobile based therary [39].

\section{Abbreviations}

PPD: Postpartum depression

EPDS: Edinburgh Postpartum Depression Scale.

\section{Data Availability}

The data used to support the findings of this study are available from the corresponding author upon request.

\section{Conflicts of Interest}

The authors declare that they have no conflicts of interest.

\section{Authors' Contributions}

Thi Kim Ly Do and Thi Thanh Huong Nguyen have the same contribution to the paper.

\section{References}

[1] C.-L. Dennis, K. Falah-Hassani, and R. Shiri, "Prevalence of antenatal and postnatal anxiety: Systematic review and metaanalysis," The British Journal of Psychiatry, vol. 210, no. 5, pp. 315-323, 2017.

[2] L. Murray, M. P. Dunne, T. Van Vo, P. N. T. Anh, N. G. Khawaja, and T. N. Cao, "Postnatal depressive symptoms amongst women in Central Vietnam: A cross-sectional study investigating prevalence and associations with social, cultural and infant factors," BMC Pregnancy and Childbirth, vol. 15, no. 1, 2015.

[3] R. Silva, K. Jansen, L. Souza et al., "Sociodemographic risk factors of perinatal depression: a cohort study in the public health care system," Revista Brasileira de Psiquiatria, vol. 34, no. 2, pp. 143-148, 2012.

[4] P. J. Surkan, K. S. Sakyi, P. Christian et al., "Risk of Depressive Symptoms Associated with Morbidity in Postpartum Women in Rural Bangladesh," Maternal and Child Health Journal, vol. 21, no. 10, pp. 1890-1900, 2017.

[5] Q. H. Vuong, "Be rich or don't be sick: estimating Vietnamese patients' risk of falling into destitution," SpringerPlus, vol. 4, no. $1,2015$.

[6] R. C. M. Ho, K.-K. Mak, A. N. C. Chua, C. S. H. Ho, and A. Mak, "The effect of severity of depressive disorder on economic burden in a university hospital in Singapore." Expert review of pharmacoeconomics \& outcomes research, vol. 13, no. 4, pp. 549559, 2013.

[7] Q. Vuong, T. Ho, H. Nguyen, and T. Vuong, "Healthcare consumers' sensitivity to costs: a reflection on behavioural economics from an emerging market," Palgrave Communications, vol. 4, no. 1, 2018.

[8] S. Liu, Y. Yan, X. Gao et al., "Risk factors for postpartum depression among Chinese women: path model analysis," BMC Pregnancy and Childbirth, vol. 17, no. 1, 2017.

[9] F. P. Tungchama, C. G. Piwuna, A. Y. Armiya'u et al., "Relationship between quality of life and postpartum depression among women in North-Central, Nigeria," Highland Medical Research Journal, vol. 17, no. 1, pp. 11-18, 2017.
[10] Y. Liu, S. Kaaya, J. Chai et al., "Maternal depressive symptoms and early childhood cognitive development: A meta-analysis," Psychological Medicine, vol. 47, no. 4, pp. 680-689, 2017.

[11] Z. Sadat, M. Abedzadeh Kalahroudi, M. Kafaei Atrian, Z. Karimian, and Z. Sooki, "The impact of postpartum depression on quality of life in women after child's birth," Iranian Red Crescent Medical Journal, vol. 16, no. 2, 2014.

[12] M. Korhonen, I. Luoma, R. Salmelin, and T. Tamminen, "A longitudinal study of maternal prenatal, postnatal and concurrent depressive symptoms and adolescent well-being," Journal of Affective Disorders, vol. 136, no. 3, pp. 680-692, 2012.

[13] C. C. Choo, K. M. Harris, P. K. H. Chew, and R. C. Ho, "What predicts medical lethality of suicide attempts in Asian youths?" Asian Journal of Psychiatry, vol. 29, pp. 136-141, 2017.

[14] F. Gressier, V. Guillard, O. Cazas, B. Falissard, N. M.-C. Glangeaud-Freudenthal, and A.-L. Sutter-Dallay, "Risk factors for suicide attempt in pregnancy and the post-partum period in women with serious mental illnesses," Journal of Psychiatric Research, vol. 84, pp. 284-291, 2017.

[15] I. Jones, P. S. Chandra, P. Dazzan, and L. M. Howard, "Bipolar disorder, affective psychosis, and schizophrenia in pregnancy and the post-partum period," The Lancet, vol. 384, no. 9956, pp. 1789-1799, 2014.

[16] C. C. Choo, P. K. Chew, C. S. Ho, and R. C. Ho, "Prediction of Quality of Life in Asian Patients with Schizophrenia: A Crosssectional Pilot Study," Frontiers in Psychiatry, vol. 8, 2017.

[17] V. Zanardo, L. Giliberti, F. Volpe, M. Parotto, F. de Luca, and G. Straface, "Cohort study of the depression, anxiety, and anhedonia components of the Edinburgh Postnatal Depression Scale after delivery," International journal of gynaecology and obstetrics: the official organ of the International Federation of Gynaecology and Obstetrics, vol. 137, no. 3, pp. 277-281, 2017.

[18] I. S. Yim, L. R. Tanner Stapleton, C. M. Guardino, J. HahnHolbrook, and C. Dunkel Schetter, "Biological and psychosocial predictors of postpartum depression: Systematic review and call for integration," Annual Review of Clinical Psychology, vol. 11, pp. 99-137, 2015.

[19] A. Fiala, J. Švancara, J. Klánová, and T. Kašpárek, “Sociodemographic and delivery risk factors for developing postpartum depression in a sample of 3233 mothers from the Czech ELSPAC study," BMC Psychiatry, vol. 17, no. 1, 2017.

[20] A. A. Alharbi and H. M. Abdulghani, "Risk factors associated with postpartum depression in the Saudi population," Neuropsychiatric Disease and Treatment, vol. 10, pp. 311-316, 2014.

[21] S. Toy, Y. Tripodis, K. Yang, J. Cook, and A. Garg, "Influence of Maternal Depression on WIC Participation in Low-Income Families," Maternal and Child Health Journal, vol. 20, no. 3, pp. 710-719, 2016.

[22] M. Sampson, Y. Villarreal, and A. Rubin, "A Problem-Solving Therapy Intervention for Low-Income, Pregnant Women at Risk for Postpartum Depression," Research on Social Work Practice, vol. 26, no. 3, pp. 236-242, 2014.

[23] A.-W. Deng, R.-B. Xiong, T.-T. Jiang, Y.-P. Luo, and W.-Z. Chen, "Prevalence and risk factors of postpartum depression in a population-based sample of women in Tangxia Community, Guangzhou," Asian Pacific Journal of Tropical Medicine, vol. 7, no. 3, pp. 244-249, 2014.

[24] A. Bauer, S. Pawlby, D. T. Plant, D. King, C. M. Pariante, and M. Knapp, "Perinatal depression and child development: Exploring the economic consequences from a South London cohort," Psychological Medicine, vol. 45, no. 1, pp. 51-61, 2015. 
[25] Z. Kozinszky and R. B. Dudas, "Validation studies of the Edinburgh Postnatal Depression Scale for the antenatal period," Journal of Affective Disorders, vol. 176, pp. 95-105, 2015.

[26] J. Gibson, K. McKenzie-Mcharg, J. Shakespeare, J. Price, and R. Gray, "A systematic review of studies validating the Edinburgh Postnatal Depression Scale in antepartum and postpartum women," Acta Psychiatrica Scandinavica, vol. 119, no. 5, pp. 350364, 2009.

[27] S. D. Shrestha, R. Pradhan, T. D. Tran, R. C. Gualano, and J. R. W. Fisher, "Reliability and validity of the Edinburgh Postnatal Depression Scale (EPDS) for detecting perinatal common mental disorders (PCMDs) among women in low-and lower-middle-income countries: A systematic review," $B M C$ Pregnancy and Childbirth, vol. 16, no. 1, article no. 72, 2016.

[28] Y. Zhao, I. Kane, J. Wang, B. Shen, J. Luo, and S. Shi, "Combined use of the postpartum depression screening scale (PDSS) and Edinburgh postnatal depression scale (EPDS) to identify antenatal depression among Chinese pregnant women with obstetric complications," Psychiatry Research, vol. 226, no. 1, pp. 113-119, 2015.

[29] C. Teo, A.-R. Chia, M. T. Colega et al., "Prospective associations of maternal dietary patterns and postpartum mental health in a multi-ethnic asian cohort: The growing up in singapore towards healthy outcomes (gusto) study," Nutrients, vol. 10, no. 3, 2018.

[30] T. V. Vo, T. K. D. Hoa, and T. D. Hoang, "Postpartum depressive symptoms and associated factors in married women: A crosssectional study in Danang City, Vietnam," Frontiers in Public Health, vol. 5, 2017.

[31] E. Bromet, L. H. Andrade, I. Hwang et al., "Cross-national epidemiology of DSM-IV major depressive episode," BMC Medicine, vol. 9, no. 1, article 90, 2011.

[32] W. Simpson, M. Steiner, M. Coote, and B. N. Frey, "Relationship between inflammatory biomarkers and depressive symptoms during late pregnancy and the early postpartum period: A longitudinal study," Revista Brasileira de Psiquiatria, vol. 38, no. 3, pp. 190-196, 2016.

[33] A. Stagnaro-Green, M. Abalovich, E. Alexander et al., "Guidelines of the American Thyroid Association for the diagnosis and management of thyroid disease during pregnancy and postpartum," Thyroid, vol. 21, no. 10, pp. 1081-1125, 2011.

[34] F. Abdollahi, M. Zarghami, M. Z. Azhar, S.-G. Sazlina, and M.S. Lye, "Predictors and incidence of post-partum depression: A longitudinal cohort study," Journal of Obstetrics and Gynaecology Research, vol. 40, no. 12, pp. 2191-2200, 2014.

[35] A. J. M. Loonen and S. A. Ivanova, "Circuits Regulating Pleasure and Happiness-Mechanisms of Depression," Frontiers in Human Neuroscience, vol. 10, no. 2016, 571 pages, 2016.

[36] A. Skalkidou, C. Hellgren, E. Comasco, S. Sylvén, and I. Sundström Poromaa, "Biological aspects of postpartum depression," Women's Health Journal (WHJ), vol. 8, no. 6, pp. 659-672, 2012.

[37] J. J. Rogathi, R. Manongi, D. Mushi et al., "Postpartum depression among women who have experienced intimate partner violence: A prospective cohort study at Moshi, Tanzania," Journal of Affective Disorders, vol. 218, pp. 238-245, 2017.

[38] E. Fitelson, S. Kim, A. S. Baker, and K. Leight, "Treatment of postpartum depression: Clinical, psychological and pharmacological options," International Journal of Women's Health, vol. 3, no. 1, pp. 1-14, 2011.

[39] M. W. Zhang, R. C. Ho, A. Loh et al., "Current status of postnatal depression smartphone applications available on application stores: an information quality analysis," BMJ Open, vol. 7, no. 11, p. e015655, 2017. 


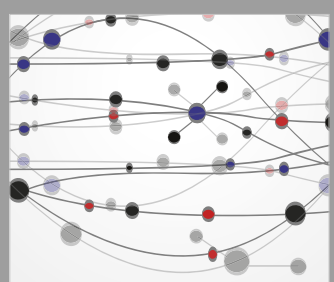

The Scientific World Journal
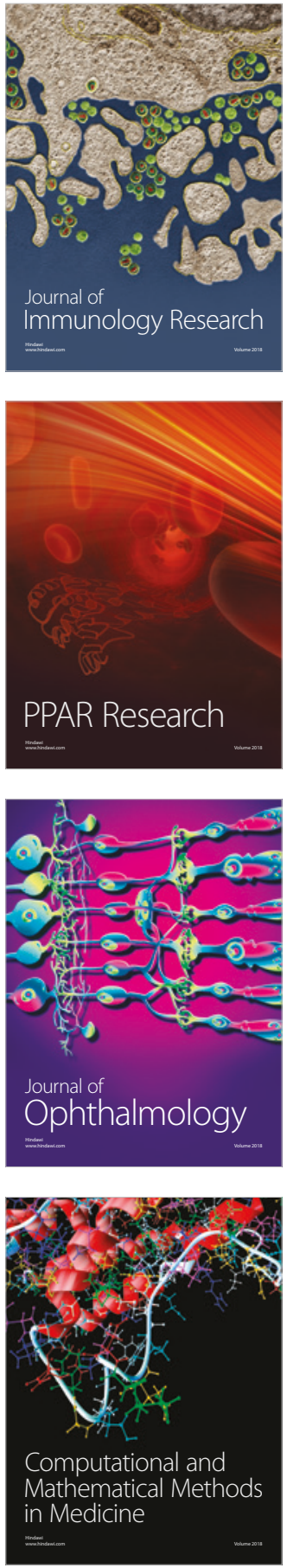

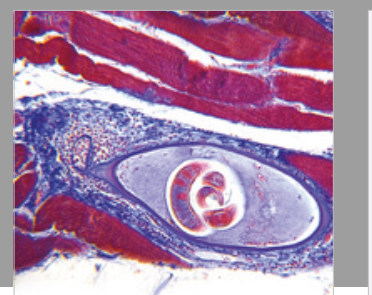

Gastroenterology Research and Practice

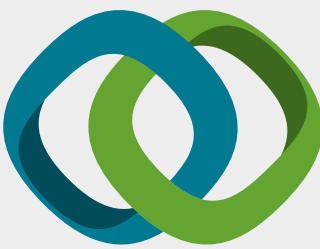

\section{Hindawi}

Submit your manuscripts at

www.hindawi.com
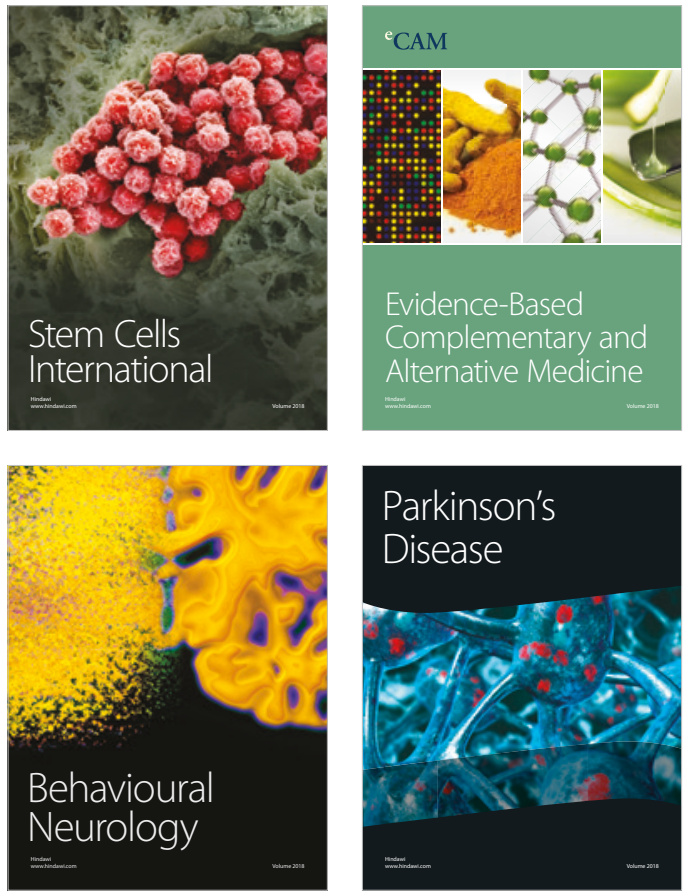

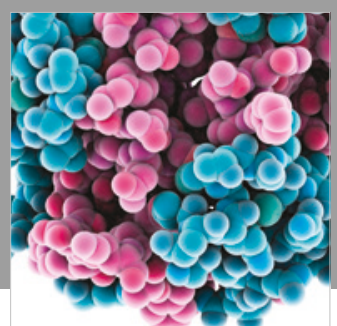

ournal of

Diabetes Research

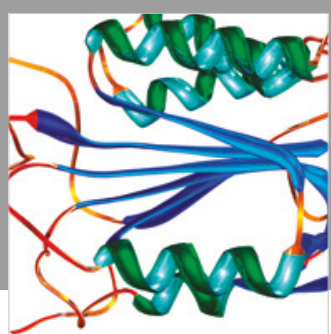

Disease Markers
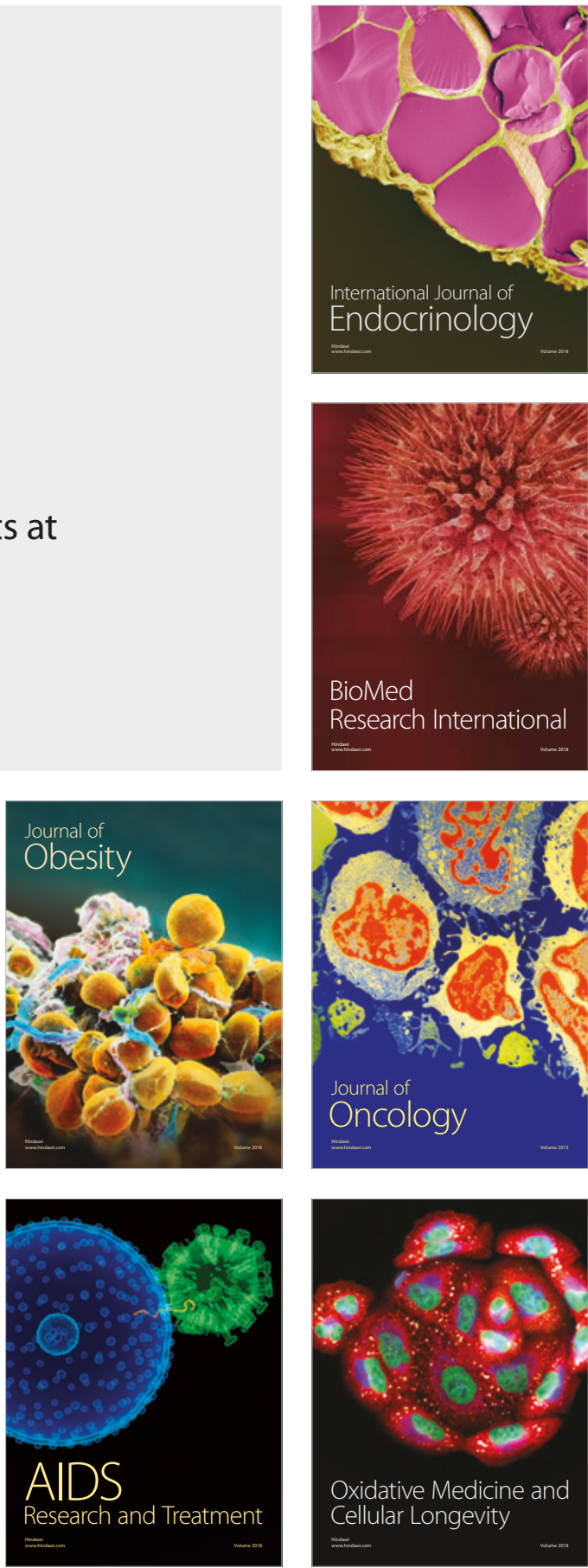